ISSN 1983-8484

Licenciado sob uma Licença Creative Commons

\title{
Contribuições da pesquisa multinível para a análise das práticas organizacionais
}

\author{
Multilevel research contributions to the organizational practices analysis
}

Samir Adamoglu de Oliveira ${ }^{[a]}$, Cristiano de Oliveira Maciel ${ }^{[\mathrm{b}]}$

[a] Doutorando em Administração pelo Programa de Pós-Graduação em Administração da Universidade Federal do Paraná (UFPR), Curitiba, PR - Brasil, e-mail: samiroliveira09@hotmail.com

[b] Doutorando em Administração pelo Programa de Pós-Graduação em Administração da Pontifícia Universidade Católica do Paraná (PPAD/PUCPR), professor da Pontifícia Universidade Católica do Paraná (PUCPR), Curitiba, PR - Brasil, e-mail: crmaciel.adm@gmail.com

\section{Resumo}

Neste ensaio tem-se como objetivo evidenciar potenciais contribuições dos delineamentos multiníveis para investigação das práticas organizacionais, apresentando-as de forma sistematizada e reflexiva. Para tanto, o trabalho trata da noção de práticas a partir da ideia de reciprocidade entre agência e estrutura social, não descolando, desse modo, a discussão sobre as atividades organizacionais que ocorrem na esfera micro dos seus condicionantes e resultados sociais. A partir da crítica ao viés das teorias macro-orientadas, que dominaram o mainstream dos estudos organizacionais até a atual "virada da prática" (practice turn) na teoria social, a ação organizacional é situada no primeiro plano dos esforços de compreensão acerca do modo como atores sociais inseridos em sistemas organizacionais acessam, de maneira negociada em suas interações, as estruturas sociais para (re)criar determinadas práticas nas organizações. Com ênfase nos aspectos de i) reciprocidade, e ii) simultaneidade entre estrutura social, sistema organizacional e atividades coletivas/individuais, foram apresentados os principais conceitos da pesquisa multinível que podem ser adotados, de maneira "traduzida", na investigação da prática organizacional.

Palavras-chave: Pesquisa multinível. Práticas. Organizações.

\footnotetext{
Abstract

The aim of this paper is to highlight the potential contributions of the multilevel design in research of organizational practices, in a systematic and reflexive way. Thus, the work shows the notion of practice from the idea of reciprocity between agency and social structure, without disarticulate the discussion about the organizational activities in the micro level from its conditions and social outcomes. From the criticism directed to macro-oriented theories bias, which dominated the mainstream of organization studies to the current practice turn in social
} 
theory, organization action is situated in the main efforts to understanding the way how social actors embedded in organizational systems access, in negotiated ways of interactions, the social structures to recreate some practices in organizations. Highlighting (i) reciprocity, and (ii) simultaneity, among social structure, organization system and collective/individual activities, it was showed the main concepts of the multilevel research that can be adopted in a "translated way" in investigation of organizational practice.

Keywords: Multilevel research. Practices. Organizations.

\section{Introdução}

0 interesse pela investigação da ação nas organizações atende à necessidade de aprofundamento acerca do termo organização como verbo, ao invés de desnecessariamente restringir seu uso como substantivo, conforme defendido por Weick (1979). Essa linha de argumentação finalmente encontra ressonância também na atual "virada da prática" (practice turn) na teoria social contemporânea (SCHATZKI; CETINA; SAVIGNY, 2001).

A teoria organizacional foi acusada de relegar por muito tempo a questão da prática, sobretudo a partir dos anos 1960, quando abordagens macro-orientadas se tornaram o caminho considerado urgente para desenvolvimento dessa área de conhecimento (MCKINLEY; MONE, 2005; STARBUCK, 2005). Na esteira do sucesso de perspectivas funcionalistas que ganharam força nessa época, como a Teoria Geral dos Sistemas, os estudos organizacionais deram espaços para contribuições evidentemente importantes, como a Teoria da Ecologia das Organizações, a Teoria da Dependência de Recursos, a Teoria da Contingência Estrutural e até mesmo a Teoria Neoinstitucional, mas ajudaram também no afastamento da investigação praxiológica dos atores organizacionais.

Em decorrência dessa ênfase nos aspectos macro, teorias baseadas em diferentes ontologias, bem como em pressupostos diferenciados de homem, características ambientais (e.g., complexidade, dinamismo, munificência, mitos e racionalidade coletiva) foram desenvolvidas particularmente para ajudar na compreensão de como fatores externos às organizações "determinavam" o seu comportamento.

Atualmente, esse pressuposto de "determinação" vem sendo gradualmente substituído pela noção de "condicionamento". Logo, adeptos das mais diferentes teorias admitem, cada vez mais facilmente, que as ações das organizações são restringidas por elementos ambientais, que, do mesmo modo, possibilitam a ação dos atores sociais. Em relação a esse ponto é importante ressaltar que essa lógica tem sido objeto de análise das principais teorias sociais contemporâneas aplicadas à análise organizacional.

Autores como Bourdieu (1990) e Giddens (2003) tentam promover a discussão sobre a interação entre práticas localizadas e estruturas sociais fazendo uso de diversas fontes de pensamento que deram vazão a muitos conceitos destinados a esclarecer de que modo as esferas macro e micro se relacionam. Assim, conceitos como habitus e estruturação são direcionados a tentar colocar em conversação elementos de ordem macro e micro. Mesmo assim, afirma-se que, embora seja crescente a concepção de que a prática organizacional é um fenômeno multinível (JARZABKOWSKI; SPEE, 2009; JARZABKOWSKI, 2004; WHITTINGTON, 2006) a operacionalização de propostas de investigação, com frequência, não considera a influência de elementos em diversos níveis sobre a prática organizacional.

Com efeito, a partir dessas reflexões, o objetivo no presente trabalho é evidenciar potenciais contribuições dos delineamentos multiníveis para investigação das práticas organizacionais, apresentando-as de forma sistematizada e discutida, sem pretensões de, com isso, iniciar um debate acerca de qual abordagem metodológica é superior ou inferior (em alusão às discussões envolvendo pesquisas qualitativas versus quantitativas). Logo, a principal contribuição do trabalho centra na "tradução" de elementos e da lógica subjacente de um método predominantemente quantitativo para aplicação em estudos com um objeto de natureza social e que, com frequência, exige o uso de técnicas qualitativas para obtenção de dados. Ao realizar-se essa "tradução", busca-se colaborar para análises que contemplem adequadamente a natureza multinível da prática social organizacional (JARZABKOWSKI; SPEE, 2009; JARZABKOWSKI, 2004; SCOTT, 2009; WHITTINGTON, 2006). 
A estrutura do trabalho está dividida em outros quatro tópicos após essa breve introdução. Inicialmente aborda-se a natureza da prática organizacional, onde definições diferenciadas de práticas e atividades são contrastadas. Na sequência, são caracterizados os delineamentos multiníveis, para posteriormente serem discutidas suas contribuições. Por último, as considerações finais são destinadas a enfatizar os principais pontos do trabalho que merecem futuras reflexões e debates.

\section{Diretrizes metodológicas}

$\mathrm{Na}$ elaboração do presente ensaio seguiram-se como diretrizes metodológicas os passos da revisão integrativa (integrative review). Esse procedimento cabe nas situações em que o pesquisador tem por objetivo sintetizar o conhecimento atual sobre determinado tópico de investigação. Assim, o primeiro passo consistiu na coleta de artigos sobre práticas organizacionais. Na sequência, esses artigos foram examinados em termos de suas similaridades e diferenças quanto ao conceito de prática, e as referências empregadas nesses trabalhos serviram de ponto de partida para a leitura de novos trabalhos. 0 próximo passo centrou na consideração de como a prática organizacional poderia ser analisada por meio de delineamentos de pesquisa multinível.

\section{A natureza da prática organizacional}

A partir de meados do século passado, uma série de pensadores e teóricos sociais começou a atentar para um elemento central para o entendimento da realidade, até então pouco explorado ou analisado de maneira tangencial em virtude das tradições de pensamento institucionalizadas, e da complexidade que envolve abordá-lo e discuti-lo: as práticas sociais. Este elemento, polissêmico e multidimensional em essência, representa para muitos um ponto de partida ontológico, de natureza social, o qual possibilita relacionar elementos explicativos da ordem das estruturas sociais mais macro às questões das atividades rotineiras e cotidianas mais micro (ORLIKOWSKI, 2010; SCHATZKI; CETINA; SAVIGNY, 2001). Assim, enfatizando interações sociais, elementos de construção coletivos em determinados tempos e espaços, e não dissociando elementos mentais de elementos corporais, tradição de criatividade, e nem aspecto rotineiro de improvisação, as práticas sociais são um elemento cuja amplitude acaba por realizar uma 'ponte' entre elementos instituídos e instituintes, conforme salienta Coulter (2001, p. 29-39, tradução nossa):

[...] a rota apropriada para a solução do problema da relação 'micro-macro' [na teoria sociológica contemporânea] deve ser encontrada mediante uma elucidação sistemática da lógica das nossas práticas comuns (incluindo nossas práticas comunicativas) nas nossas circunstâncias comuns [...] uma apreciação adequada no papel da 'macro-ordem' nas nossas vidas cotidianas revela que tais fenômenos são variavelmente instanciados mediante aquilo que nós dizemos e fazemos e também que nossas condutas são "onirelevantemente" relacionadas às considerações do 'macro-nível'.

Segundo Strati (2007, p. 65), as práticas têm total relação com as questões do conhecimento humano nos planos mais amplos da vivência cotidiana, sob aspectos conscientemente sistematizáveis ou não. Para este autor, as práticas compreendem: i) o conhecimento situado a fenômenos locais, mas ao mesmo tempo conectado aos planos mais amplos; ii) a questão da "performatividade" nas ações humanas, considerando as interações humanas e não humanas como elementos inter-relacionados de maneira tácita e não tácita; iii) os elementos materiais culturais, artefatos e espaços nos quais se manifestam pervasivamente práticas laborais, discursivas e códigos normativos; e iv) o elemento da legitimidade para a aceitação e sustentação social das práticas em si.

Dessa forma, a sociomaterialidade das práticas se faz pervasivamente vivenciável em contextos interacionais a partir das cadeias e nexus de ação que se entrelaçam e atravessam os fazeres e dizeres do dia a dia, conferindo nuances, maneirismos e naturezas particulares às inúmeras práticas sociais que um determinado grupo pode sustentar no seu cotidiano, abarcando, assim, elementos racionais e não racionais instanciados na tradição, mas passíveis de (re)construção a partir da capacidade de agência dos sujeitos que as acessam e as manifestam (SCHATZKI, 1996, 2001).

Conforme Chia e MacKay (2007), a natureza "transindividual" que as práticas sociais possuem é o elemento central para se entender como elas orientam e educam a nossa atenção e moldam as nossas 
disposições na vivência diária, já que tanto a natureza social que construímos e reconstruímos nas nossas ações quanto os processos que criamos e fazemos uso para esse fim são subordinados a e constituídos por práticas e complexos de práticas, de maneira tanto consciente quanto subconsciente, ao mesmo tempo. Isso implica a compreensão de que, muito mais do que um elemento objetivo além do nosso espectro de existência - o que denotaria perguntar qual a nossa relação com as práticas -, as práticas sociais estão presentes em nós mediante o que fazemos e dizemos, contemplando planos racionais, emocionais, laborais, etc.

É nesse sentido, de que os seres humanos não são seres de racionalidade plena, mas, muito pelo contrário, que abarcam em sua natureza elementos de diversas sortes (intelectuais, comportamentais, analíticos, emocionais, etc.), que se tem, contemporaneamente, um desenvolvimento de "visões baseadas em práticas sociais" que visam analisar fenômenos e processos da vivência humana cotidiana em inúmeros contextos sociais, a exemplo das organizações e daquilo que nelas acontece diariamente.

0 que é valido frisar nessas visões baseadas em práticas é o fato de que elas necessitam não ser desatreladas de explicações envolvendo estruturas cognitivas, lógicas institucionais e elementos fundamentais que constituem o tecido social no qual elas (as práticas sociais) estão fincadas - a exemplo de crenças, valores, racionalidades, etc. Essa preocupação é crucial para um entendimento rico e mais verossímil da realidade dos agentes em contextos de análise (como as organizações, por exemplo), pois, do contrário, pode-se desenvolver argumentos tautológicos, nos quais a pura explicação "da prática pela prática" seria o suficiente para se analisar e se entender como a realidade é continuamente (re)construída no seu cotidiano (MARSHALL, 2008).

Ao circunscrever-se essa noção de prática social ao ambiente das organizações, passa-se a ter de considerar uma série de características pronunciadas em maior ou menor grau, de acordo com cada realidade analisada, a citar: i) a brevidade e a variedade das suas atividades intrínsecas; ii) a fragmentação e a descontinuidade de suas tarefas; iii) a orientação para a ação (laboral e comunicativa); iv) o favorecimento das maneiras formais e informais de comunicação; v) a natureza pervasiva e hierarquizada das suas atividades; vi) a velocidade e o ritmo implacável das suas atividades; vii) o controle das atividades realizado de maneira mais sutil do que explícito (MINTZBERG, 2009).
Dessa forma, o que se faz necessário é um delineamento mínimo versando tal conceito para a realidade organizacional, tendo em vista que uma prática social se estende além das fronteiras organizacionais, permeando, naturalmente, os universos particulares dos indivíduos nas esferas sociais de suas existências. Dessa forma, "práticas organizacionais" podem ser entendidas como sendo:

[...] ações sociais regularizadas e recorrentes que continuamente constroem e reconstroem a organização como um sistema social espaciotemporalmente delimitado. São, portanto, práticas sociais que ocorrem no contexto organizacional (ALBUQUERQUE FILHO; MACHADO-DA-SILVA, 2009, p. 632).

As práticas manifestam formas de pensar investidas em formas de agir, que arranjam e rearranjam elementos distintos entre si (num processo de bricolagem), resultando numa "arte de combinação a qual não pode ser dissociada de uma arte de utilização" (DE CERTEAU, 2002, p. xv, tradução nossa), nem sempre voluntária e consciente, dada a multiplicidade de estruturas existentes que acessamos e que se investem sobre nós, sendo impossível, portanto, o pleno conhecimento e clareza dessa pluralidade (DE CERTEAU, 2002; GIDDENS, 2003; SCHATZKI, 2001). Ao realizar-se pesquisa social em organizações, todos esses elementos de pluralidade e complexidade que as práticas sociais abarcam passam a ser considerados juntamente com forças condicionantes tanto do nível micro inerente à organização quanto de nível macro, externos àquela, envolvendo questões culturais, institucionais, políticas, tecnológicas, econômicas, dentre outras (SCOTT, 2009).

\section{Delineamentos multiníveis}

0 desenvolvimento de uma abordagem multinível para a pesquisa organizacional visa captar a complexidade da realidade social das organizações, convencendo pesquisadores sociais de que a questão dos níveis que existem em uma organização precisa não apenas estar contemplada na realização das pesquisas, mas também discutido e analisado em todo o processo de desenho de uma pesquisa.

Essa intenção (e também "preocupação") reside no pressuposto de que as organizações são sistemas sociais com múltiplos níveis constitutivos, mas que, 
por razões da própria evolução dos estudos e da sua pesquisa, passou a ser desconsiderado, quando não minimizado e/ou esquecido pelos pesquisadores, frente às ambiguidades e descuidos interpretativos destes. Assim, a proposta de uma abordagem multinível também reside no resgate do valor heurístico desses níveis para a análise e entendimento de uma organização (KOZLOWSKI; KLEIN, 2000).

Subjacente a essa questão, há uma problemática que permeia os estudos organizacionais como um todo (e que também é herança da evolução das correntes de pensamento que nutrem e nutriram este campo): a relação entre perspectivas macro (de raízes sociológicas, preocupadas com regularidades no comportamento social que transcendem as aparentes diferenças entre os seus atores - numa lógica top-down para esses efeitos) e as perspectivas micro (cujas raízes na psicologia sugerem que há variações no comportamento individual que precisam ser consideradas em si mesmas, no que tange à sua importância para a explicação de fenômenos sociais - numa lógica bottom-up para esses resultados).

A partir disso, abre-se uma 'janela' para um caminho mais integrador entre essas perspectivas (algo como uma perspectiva meso de análise) (BRASS, 2000), o qual é apreendido, em ideia, a partir de uma teoria "formativa", ou seja, de uma teoria que passe a considerar a importância das interações em seu plano mais micro, alicerçando as bases para que as interações não apenas em outros níveis, mas também as interações entre esses outros níveis ganhem mais sentido. Nisso, passa a ficar claro que pensar o micro e o macro não é necessariamente pensar numa lógica multinível; este último significa, acima de tudo, pensar as interações dentro de e entre níveis, fomentando as explicações para os fenômenos organizacionais (KOZLOWSKI; KLEIN, 2000).

Kozlowski e Klein (2000) frisam que há de se considerar princípios centrais em torno dos quais a lógica multinível precisa estar fincada. Estes são:

- no que focar e pesquisar (what)?

- como relacionar fenômenos entre níveis diferentes de análise (how)? Onde se originam e onde culminam tais fenômenos/processos entre níveis (where)?

- quando ocorrem tais fenômenos e suas relações (when)?

- por que esses fenômenos e as interações entre seus diferentes níveis ocorrem, e por que não ocorrem de outra forma, ou melhor, por que o pesquisador optou por analisá-los dessa forma, e não de outra, na construção da sua pesquisa e do seu argumento teórico (why e why not)?

Para todos esses pontos, Kozlowski e Klein (2000) elencam uma série de "princípios" analíticos, que longe de serem sugeridos como "leis", figuram apenas como nortes para se pensar tais questões.

É interessante perceber que, permeando todas essas questões, está uma lógica integradora por si própria, como a de não dissociar tempo de espaço (where e when), ou ainda a de combinar descrição com exposição analítica (what e how). Além destes, também é interessante notar a cautela em se contemplar possibilidades integradoras também para as implicações de corte temporal da pesquisa, associando lógicas transversais (para fenômenos mais macro, ou de lógica top-down) com fenômenos de lógicas longitudinais (para fenômenos mais micro, cuja natureza emergente remete à lógica bottom-up). Um exemplo disso é a explicação de reverberação ou "arrastamento" de um processo de emergência de um fenômeno ao longo do tempo (entrainment), a partir do qual os seus efeitos são sentidos nos outros níveis analisados em uma organização (e/ou além dela) à medida que este se estende ao longo de um dado tempo e um dado espaço.

Diante disso, é fundamental a relação (justaposta, ou seja, não mutuamente excludente) entre uma característica de "composição" na emergência de um fenômeno (de implicações mais homogeneizantes) e característica de "compilação" na emergência de um fenômeno (de implicações mais heterogeneizantes) duas concepções de natureza bottom-up, conforme discutidas numa lógica multinível.

Os processos de compilação baseiam-se na premissa de que os fenômenos constituem uma área de domínio comum que perpassa vários níveis, mas que existem diferenças entre quando os dados são agregados e quando os dados não são agregados. De tal sorte, nos modelos de compilação, espera-se que dados coletados em nível mais baixo variem dentro dos grupos; já quando os dados são agregados, espera-se que a variável agregadora seja capaz de medir algum fenômeno que não esteja evidente no nível mais baixo. Em geral o pesquisador não precisa se preocupar em estabelecer concordância ou demonstrar a confiabilidade da média de grupo para estabelecer a validade de construto de medidas agregadas baseadas na compilação (BLIESE, 2000). 
O oposto dos processos de compilação, os processos de composição baseiam-se na premissa de que fenômenos de nível mais baixo são isomórficos uns com os outros e com o construto de nível mais alto. Nos modelos de processos de composição assume-se que as variáveis agregadoras são essencialmente idênticas aos construtos de nível mais baixo, tanto em sua forma quanto em sua função. Não obstante à habitual dependência teórica em modelos de composição em pesquisa organizacional, o isomorfismo verdadeiro é raro. Em vez disso, pesquisadores têm uma probabilidade maior de encontrar isomorfismo parcial, no qual a variável agregadora mantém ligações conceituais com a variável de nível mais baixo, ainda que ela seja bastante distinta (BLIESE, 2000).

Bliese (2000) chama de processos de composição nebulosa (fuzzy) aqueles processos bottom-up nos quais a agregação de construtos de nível mais baixo em variáveis de nível mais alto tende a criar uma variável de nível agregado que é simultaneamente relacionada com, e diferente de sua equivalente de nível mais baixo.

Em ambos, os processos de composição nebulosa e os processos de compilação, a variável agregadora é considerada não isomórfica com sua equivalente de nível mais baixo. Apesar dessa semelhança, eles são processos bem diferentes. Nos processos de compilação a variável agregadora é diferente de sua equivalente em termos qualitativos, enquanto nos processos de composição nebulosa a variável agregadora mantém ligações próximas com sua equivalente de nível mais baixo, mas de forma sutil e diferente.

Já no que diz respeito aos princípios que especifiquem a formulação de modelos de pesquisa que levem em conta a lógica multinível, Kozlowski e Klein (2000) consideram basicamente três elementos: unidades globais (global); unidades compartilhadas (shared); e unidades configuracionais (configurational), todas estas levando-se em conta as propriedades que cada uma dessas unidades contém (ou devem conter).

A primeira, de natureza global, suscita a formulação de construtos de pesquisa com características objetivas, descritivas e facilmente observáveis, na qual o elemento de "composição" (ou seja, o elemento homogeneizante) se faz mais notável.

A segunda, de natureza compartilhada, já compreende características que são comuns aos membros do nível observado, sem serem, contudo, facilmente observáveis e/ou identificáveis. Mesmo sustentando esse elemento mais de "latência" (digamos assim), essas unidades e suas propriedades ainda são dependentes de elementos de "composição".

Já a terceira, de natureza configuracional, corresponde a arranjos ou padrões que não necessariamente convergem ou coincidem em suas essências, tendo, portanto, mais associação com a apreensão da diferença, remetendo ao elemento da "compilação".

No que concerne aos fenômenos emergentes (os fenômenos bottom-up), é crucial notar que este tem origem em comportamentos, cognição, agregados sensíveis e viscerais, e outras características dos indivíduos, e que são amplificados pelas interações entre estes, para então se manifestarem como um fenômeno coletivo. A tipologia de emergência proposta por Kozlowski e Klein (2000) justapõe composição e compilação e inclui os seguintes tipos de emergência: emergência convergente, emergência combinada forçada, emergência combinada espontânea, emergência mínimo/máximo, emergência variante e emergência padronizada. Cada um desses tipos descreve um processo de emergência distinto, baseado em restrições contextuais e processos de interação, que busca explicar como um fenômeno oriundo de um nível inferior se manifesta em um nível superior. A tipologia contribui para elaborar o suporte teórico que molda a conceitualização de formas alternativas de emergência.

A partir desses conceitos, tanto Kozlowski e Klein (2000) quanto Bliese (2000) defendem que a perspectiva multinível fornece meios para os pesquisadores unificarem a ciência social e criarem uma base sólida para melhorar a análise do impacto de práticas para disciplinas que estudam organizações.

\section{Contribuições dos delineamentos multiníveis para o estudo da prática}

As principais características da pesquisa multinível que podem ser consideradas na investigação da prática organizacional e de onde são extraídas suas principais contribuições são: (i) separação de níveis, (ii) isolamento da influência de características de diversos níveis sobre outras variáveis, (iii) consideração de processos topo-base (top-down), mas também base-topo (bottom-up), (iv) identificação de variação entre e dentro de diferentes grupos, (v) consideração tanto de processos composicionais (isomórficos) quanto de processos compilatórios (modelo de descontinuidades), (vi) ajustamento temporal das pesquisas que consideram processos de emersão, ou base-topo (bottom-up). 
Separação de níveis

A separação de níveis, mais recorrente sobretudo na sociologia contemporânea, é a localização hierárquica da prática a partir de um conceito dual. Prática significa tanto algo que guia o que atores sociais fazem (macro) quanto atividades executadas no plano material (micro) (WHITTINGTON, 2006). Contudo, vale ressaltar que a separação de níveis não passa de uma divisão de interesse teórico, conforme Klein e Kozlowski (2000). Assim, limites entre um e outro sistema são produtos da (re)produção na ação (enactment) dos seus observadores. Logo, é possível argumentar aqui que existem muitos níveis macros e micros naquilo que é definido por "micro" nas teorias sociais contemporâneas que alcançam maior ressonância nos estudos organizacionais, contemplando assim possibilidades de comparativos analíticos entre níveis diferentes, respeitando o dinamismo de uma realidade social, como a organizacional (SCOTT, 2009).

Isolamento da influência de características de diversos níveis

Ao observar a prática, é preciso considerar que ela pode ser ao mesmo tempo variável dependente e independente ao ser tipologizada em diferentes clusters de atividades recorrentes dentro ou fora das organizações. Fora da organização significa práticas de associações, concorrentes, fornecedores, clientes, consultorias e escolas de negócios que exercem influência sobre o que ocorre dentro das organizações (MACHADO-DA-SILVA; VIZEU, 2007). Essas seriam práticas que poderiam ser segmentadas em níveis diferentes: ao abraçar elementos como concorrentes, associações, fornecedores, clientes e consultorias como atores sociais no nível macro das organizações e outros elementos organizacionais constituintes dos níveis meso e micro, abrem-se várias oportunidades para considerar a influência de atributos em diversos níveis.

\section{Consideração de processos topo-base e base-topo}

Outros conceitos importantes que foram sistematizados pela abordagem multinível fazem referência ao modo de relacionar diferentes níveis. Esses processos são classificados em: processos topo-base (top-down) e base-topo (bottom-up). Essa classificação permite que sejam consideradas analiticamente as duas fases de uma lógica recursiva, tanto a influência de características macro quanto a emersão de fenômenos que se dá no nível micro. A partir dessa noção, abre-se uma via de análise que faz jus às possibilidades de entendimento de influências processuais nas práticas tanto por meios intraorganizacionais quanto por forças extraorganizacionais (provenientes do campo, do setor, da população, do nível societal, etc.), conferindo "camadas de elementos explicativos" do contexto ao qual pertence $(\mathrm{m}) \mathrm{a}(\mathrm{s})$ organização(ções) a análise (CARONNA; POLLACK; SCOTT, 2009, p. 266).

Identificação de variação entre e dentro de diferentes grupos

Um pressuposto deveras importante na lógica multinível é o de que os processos topo-base e base-topo devem considerar a existência de diferentes grupos dentro das organizações, bem como fora delas, ou seja, em outros níveis com variações em suas práticas. Como afirmam Kozlowski e Klein (2000), seguindo outros autores, a diferenciação, ou seja, a não uniformidade marca mais acentuadamente os fenômenos organizacionais do que o isomorfismo. Tal assertiva é válida também para as práticas organizacionais. Diferentes grupos de indivíduos, e até mesmo indivíduos dentro de uma organização apresentam heterogeneidade em suas práticas, quer essa diferença seja material ou nos modos de acessar uma mesma atividade material (DE CERTEAU, 2002).

As práticas dos indivíduos nas organizações se conformam em rotinas com execução efetivamente compartilhada entre uma comunidade de atores organizacionais, mas não necessariamente compartilhada entre todos os grupos. Se essas práticas são realizadas por indivíduos e grupos, é natural pensar que elas apresentem variação entre diferentes grupos. 0 pressuposto da variação dentro e entre grupos força o pesquisador da prática organizacional a dirigir seu olhar também para as características de diferentes níveis (indivíduo, grupo informal, departamento, unidade) que influenciam a prática em análise, preservando, assim como mencionado para outros pressupostos dos delineamentos multiníveis, a complexidade da realidade social (LACEY; FISS, 2009). 
Consideração tanto de processos

composicionais quanto compilatórios

E, ao considerar a variação dentro e entre diferentes grupos em relação a uma mesma prática, surge a necessidade de esclarecer que processos topo-base e base-topo podem dar-se de modo composicional ou compilatório. Isso significa que características de diferentes níveis podem ser de inclinação mais isomórficas ou mais heterogêneas. Isso é particularmente importante nos casos de processos de emersão (bottom-up). No caso de transformações de práticas que são dadas como institucionalizadas em níveis superiores e assumidas como isomórficas, muitas delas têm início na diferenciação entre as características internas de uma organização, grupos e departamentos que são influenciados por fatores tais como: competição, relações de poder, conflito, formação de coalizões e desacordos. Desse modo, as práticas que estão presentes em uma organização têm sua emersão marcada por várias interações e negociações, sendo possível apreender a sua formação apenas via a consideração de processos compilatórios com direção base-topo (bottom-up).

\section{Ajustamento temporal das pesquisas que} consideram processos de emersão

Outro importante fator que a pesquisa multinível chama a atenção dos pesquisadores da prática organizacional é a escala de tempo dos processos topo-base e base-topo. Processos de emersão (base-topo) apresentam tempos maiores de realização. Desse modo, estudos sobre práticas organizacionais que contemplam processos topo-base teriam maiores chances de sucesso no desenvolvimento de estudos transversais ou de curto prazo, mesmo na adoção de uma "perspectiva" longitudinal de avaliação. Por outro lado, pesquisadores que assumam a existência de um processo de emersão (base-topo) precisam ampliar acentuadamente seus horizontes de tempo de investigação.

Vantagens do estudo da prática inspirado na pesquisa multinível

Por fim, é possível advogar que estudos acerca das práticas organizacionais que estejam inspirados nos principais conceitos da pesquisa multinível, conforme discutidos anteriormente, podem alcançar as seguintes vantagens em comparação aos estudos que adotam uma perspectiva de um único nível:

a) contribuem para o desenvolvimento e melhor especificação de teorias que consideram efeitos de múltiplos níveis sobre alguma variável dependente;

b) exigem definir que práticas estão localizadas nos níveis micro, meso e macro dentro daquilo que se convencionou rotular apenas como nível micro pelas teorias sociais;

c) permitem a visualização de procedimentos que isolem parcialmente alguns elementos explicativos, para fins de facilitação e sistematização não apenas da pesquisa, mas sobretudo da análise, ao reconhecerem a existência de processos topo-base, mas também processos base-topo;

d) permitem considerar a variação de práticas e seus antecedentes entre e dentro dos grupos, levando o pesquisador a encarar que, com frequência, as práticas organizacionais são mais grupais e individuais do que uma totalidade coletiva, preservando a complexidade subjacente a esse elemento de análise na pesquisa social em organizações;

e) direcionam o olhar do pesquisador para a classificação da natureza da reprodução ou formação das práticas ao considerar a existência de processos composicionais e compilatórios;

f) contribuem na explicação da necessidade de maior alcance temporal dos estudos sobre a prática, particularmente no caso da investigação de processos emergentes, que ocorrem de forma mais cadenciada em comparação aos processos topo-base.

\section{Considerações finais}

0 presente ensaio teve como proposta discutir de que maneira os princípios e conceitos centrais da pesquisa multinível podem contribuir no delineamento de estudos sobre a prática organizacional, predominantemente investigado via procedimentos qualitativos.

A reflexão sobre a aplicação desses princípios e lógicas de pesquisa trouxe à tona uma série de justificativas, explicações e aproximações entre os pontos de partida dos métodos quantitativos e qualitativos. Foi possível 
verificar como alguns dos desafios impostos aos pesquisadores qualitativistas das práticas são enfrentados, também, por pesquisadores que adotam procedimentos estritamente de análise de dados quantitativos.

No decorrer do texto foram considerados como conceitos e princípios centrais da perspectiva multinível as seguintes características: (i) separação de níveis, (ii) isolamento da influência de características de diversos níveis sobre outras variáveis, (iii) consideração de processos topo-base (top-down), mas também base-topo (bottom-up), (iv) identificação de variação entre e dentro de diferentes grupos, (v) consideração tanto de processos composicionais (isomórficos) quanto de processos compilatórios (modelo de descontinuidades), (vi) ajustamento temporal das pesquisas que consideram processos de emersão, ou base-topo (bottom-up). Essas características podem encontrar ressonância nos desafios dos pesquisadores da práxis organizacional, trazer justificativas para escolhas metodológicas, ou mesmo apontar a necessidade de reflexões sobre as atuais escolhas desses pesquisadores.

Além dessas questões, o presente ensaio colabora para as discussões metodológicas acerca das investigações sobre práticas nas organizações, juntamente com outras possibilidades de estudos multiníveis, de cunho mais qualitativo, como métodos qualitativos comparativos (CARONNA; POLLACK; SCOTT, 2009; LACEY; FISS, 2009; RAGIN, 2008), diante da atual demanda por estudos centrados na problemática das práticas sociais como rota para a superação de dualismos que, pervasivamente, marcaram presença nos estudos sociais há décadas.

\section{Referências}

ALBUQUERQUE FILHO, J. B.; MACHADO-DA-SILVA, C. L. Práticas organizacionais e estrutura de relações no campo do desenvolvimento metropolitano. Revista de Administração Contemporânea, v. 13, n. 4, p. 626-646, 2009.

BLIESE, P. D. Whithin-group agreement, non-independence, and reliability: implications for data aggregation and analysis. In: KLEIN, K. J.; KOZLOWSKI, S. W. J (Ed.). Multilevel theory, research, and methods in organizations - foundations, extensions and new directions. San Francisco, CA: Jossey-Bass Inc., 2000. p. 349-381.

BOURDIEU, P. The logic of practice. Cambridge: Polity Press, 1990.
BRASS, D. J. Networks and frog ponds: trends in multilevel research. In: KLEIN, K. J.; KOZLOWSKI, S. W. J. (Ed.). Multilevel theory, research, and methods in organizations - foundations, extensions and new directions. San Francisco, CA: Jossey-Bass Inc., 2000. p. 557-571.

CARONNA, C. A.; POLLACK, S. S.; SCOTT, W. R. Organizations, populations, and fields: investigating organizational heterogeneity through a multilevel case study design. Research in the Sociology of Organizations, v. 26, p. 249-270, 2009.

CHIA, R.; MACKAY, B. Post-processual challenges for the emerging strategy-as-practice perspective: discovering strategy in the logic of practice. Human Relations, v. 60, n. 1, p. 217-242, 2007.

COULTER, J. Human practices and the observability of the 'macro-social'. In: SCHATZKI, T. R.; KNORR CETINA, K.; SAVIGNY, E. von (Ed.). The practice turn in contemporary theory. London: Routledge, 2001.

DE CERTEAU, M. The practice of everyday life. Berkeley, LA: University of California Press, 2002.

GIDDENS, A. A constituição da sociedade. São Paulo: Martins Fontes, 2003.

JARZABKOWSKI, P. Strategy as practice: recursiveness, adaptation, and practices-in-use. Organization Studies, v. 25 , n. 4 , p. $529-560,2004$.

JARZABKOWSKI, P.; SPEE, A. P. Strategy-as-practice: a review and future directions for the field. International Journal of Management Review, v. 11, n. 1, p. 69-95, 2009.

KOZLOWSKI, S. T. J.; KLEIN, K. J. A multilevel approach to theory and research in organizations: contextual, temporal, and emergent processes. In: KLEIN, K. J.; KOZLOWSKI, S. W. J. (Ed.). Multilevel theory, research, and methods in organizations - foundations, extensions and new directions. San Francisco, CA: Jossey-Bass Inc., 2000, p. 3-90.

LACEY, R.; FISS, P. C. Comparative organizational analysis across multiple levels: a set-theoretic approach. Research in the Sociology of Organizations, v. 26, p. 91-116, 2009.

MCKINLEY, W.; MONE, M. A. Micro and macro perspectives in organization theory. In: TSOUKAS, H.; KNUDSEN, C. (Org.). The Oxford handbook of organization theory. Oxford: Oxford University Press, 2005. p. 345-372.

MACHADO-DA-SILVA, C. L.; VIZEU, F. Análise institucional de práticas formais de estratégia. Revista de Administração de Empresas, v. 47, n. 4, p. 89-100, 2007. 
MARSHALL, N. Cognitive and practice-based theories of organizational knowledge and learning: incompatible or complementary? Management Learning, v. 39, n. 4, p. 413-435, 2008.

MINTZBERG, H. Managing. San Francisco: Berrett-Koehler, 2009.

ORLIKOWSKI, W. J. Practice in research: phenomenon, perspective and philosophy. In: GOLSORKHI, D.; ROULEAU, L.; SEIDL, D.; VAARA, E. (Ed.). Cambridge handbook of strategy as practice. Cambridge: Cambridge University Press, 2010. p. 23-33.

RAGIN, C. C. Redesigning social inquiry: fuzzy sets and beyond. Chicago: University of Chicago Press, 2008.

SCHATZKI, T. R. Social practices: a wittgensteinian approach to human activity and the social. Cambridge: Cambridge Universitiy Press, 1996.

SCHATZKI, T. R.; CETINA, K. K.; SAVIGNY, E. von. The practice turn in contemporary theory. London: Routledge, 2001.

SCHATZKI, T. R. Practice mind-ed orders. In: SCHATZKI, T. R.; KNORR CETINA, K.; SAVIGNY, E. von (Ed.). The practice turn in contemporary theory. London: Routledge, 2001. p. $42-55$.
SCOTT, W. R. Comparing organizations: empirical and theoretical issues. Research in the Sociology of Organizations, v. 26, p. 45-62, 2009.

STARBUCK, W. H. The origins of organization theory. In: TSOUKAS, H.; KNUDSEN, C. (Org.). The Oxford handbook of organization theory. Oxford: Oxford University Press, 2005. p. 143-182.

STRATI, A. Sensible knowledge and practice-based learning. Management Learning, v. 38, n. 1, p. 61-77, 2007.

SZTOMPKA, P. Society in actions. Cambridge: Polity Press, 1991.

WEICK, K. E. The social psychology of organizing. Reading, MA: Addison-Wesley, 1979.

WHITTINGTON, R. Completing the practice turn in strategy research. Organization Studies, v. 27, n. 5, p. 613-634, 2006.

Recebido: 04/07/2011

Received: 07/04/2011

Aprovado: 29/07/2011

Approved: 07/29/2011 